\title{
11 year variation in third harmonics of cosmic ray intensity on quiet days
}

\section{M.K. Richharia}

Department of Physics

Govt. Model Science College (Autonomous) Jabalpur (M.P.) India

(E-mail: mkrichharia@yahoo.com)

Presently at: Govt. R.D.College Mandla (M.P.) India

The aim of this work is to study the long term variation in third harmonics of cosmic ray intensity on sixty quietest days in a year using the data of high latitude and low latitude neutron monitoring stations during the solar cycle 20-22. It has been observed that in spite of the abrupt change in the amplitude and phase of third harmonic of cosmic ray intensity, the amplitude of third harmonics is relatively larger during the solar cycle 21 as it is observed during the declining phase of earlier solar cycle 20.Therefore this enhancement explicit point out 11 year periodicity in third harmonics of cosmic ray intensity at equatorial neutron monitoring stations. Further, the phase shift to later hours during the year 1990 showing the dependence on the polarity of solar magnetic field, which is attributed to drift effect at high latitude neutron monitoring station

Key words: Cosmic Ray/Gamogenetically quiet days/ Higher harmonics. 


\section{Introduction}

The anisotropic variation of galactic cosmic rays and their characteristics are studied through the diurnal, semi-diurnal, Tri-diurnal component mainly and the level of the isotropic intensity collectively provides fingerprint for identifying the modulating process and the electromagnetic state of interplanetary space in the neighborhood of the Earth. Many workers have attempted to derive relationship between the mean daily variation and the level of solar and geomagnetic activity. [1] The spatial anisotropy of the galactic cosmic ray intensity in the interplanetary space manifests itself as daily variation with a period of 24 hours (and its higher harmonics) due to the rotation of the Earth in the course of a day. The power Spectrum analysis as well as the Fourier analysis of the long term data of the 24-hour values of comic rays (CR) intensity observed by Earth based detectors have provided daily variation of extraterrestrial origin [2-4]. However, the amplitude of the fourth harmonics is still controversial [5-7]. Moreover, it has been observed that the amplitude and phase of tri diurnal anisotropy of CR intensity on quiet days vary considerably from one period to another. On the long term behaviour of the first three harmonics showed that high degree of year to year variability exists, a trend with solar activity was evident. The studies of the higher harmonics in the daily variation of cosmic ray provided valuable information as to the nature of the cosmic ray modulation in the heliosphere.

\section{Analysis of the data}

Solar daily variation has been studied in terms of helio-magnetic activity. A new concept of data analysis has been introduced for studying the long/short term daily variation in CR intensity recorded with neutron monitors. Fourier technique has been applied on different types of group of days chosen according to their different geomagnetic condition.

1. All days: This means all the $365 / 366$ days in year. Thus, these days are termed as AD. Of course ignoring the days with abrupt changes.

2. Quiet days: Those days on which the transient magnetic variation are regular and smooth are said to be magnetically quiet or Q days. The criteria is based upon Ap and Kp values. There are two types of days.

(i) 60 Quiet days: According to solar geophysical data (SGD) lowest mean order number are the five quietest days in a month. Thus, $60 \mathrm{Q}$ days in a year; termed as 60 QD.

(ii) 120 Quiet days: First ten quiet days in a month. Thus, 120 Q Days in a year; termed as 120 Quiet days. 
The pressure corrected hourly CR intensity data (corrected for meteorological effects) on geo magnetically five quietest days (QD) in every month for Tokyo (Lat: $35.75^{\circ} \mathrm{N}$; Cutoff rigidity; $11.61 \mathrm{GV}$; Longitude; $139.72^{\circ} \mathrm{E}$; Altitude; 20m), Mount Nourikura (Lat: $36.12^{\circ} \mathrm{N}$; Cutoff rigidity; $11.39 \mathrm{GV}$; Longitude; $137.56^{\circ} \mathrm{E}$; Altitude; $27.7 \mathrm{~m}$ ) and Inuvik (Lat: $68.35^{\circ} \mathrm{N}$; Cutoff rigidity; $0.18 \mathrm{GV}$; Longitude; $226.27^{\circ} \mathrm{E}$; Altitude; $21 \mathrm{~m}$ ) neutron monitoring stations for the period 1978-94, have been used in Fourier analysis. After applying the trend corrections, such a set of data have been subjected to Harmonic analysis for each day [8]. The average values of the amplitude and phase (local tine of the station) of the third (tri diurnal) harmonics on yearly basis have been obtained. According to solar geophysical data five quietest days are selected in a month; thus 60 quietest days are obtained in a year. These days are called international quiet days (QD). The days with extra ordinary large amplitude, if any, have not been considered. Further, the variation in the tri-diumal anisotropy with the reversal of polarity of solar magnetic field (PSMF) on 60 QD has been also investigated. Also all those days are discarded having more than three continuous hourly data missing.

\section{Result and discussion}

The yearly average amplitude and the phase of the third harmonics of daily variation for Inuvik, Tokyo and Mount Nourikura Neutron Monitoring Station during the period 198090 have been plotted in Fig. 1 on QD. It is quite apparent from Fig. 1 that there is no systematic change in the amplitude of third harmonics on QDs. Nevertheless, the amplitude of the third harmonics on QD remains relatively large during the declining phase of solar cycle 21 and 22 as compared with the declining phase of the earlier solar cycle 20 at equatorial stations [13]. The enhancement explicity point out the 11 year periodicity $[14,15]$. Therefore, it is clear that the 11 year variation in third harmonics of CR intensity due to the polarity of solar magnetic cycle. Further, the amplitude of the third harmonics of daily variation on QD is observed to be significantly low during the year 1981, which coincides with phase reversals of the solar poloidal magnetic field. Furthermore, amplitude of the third harmonics on QD has low values during minimum solar activity period.

The amplitude of third harmonics of daily variation in the year 1980 has quite abruptly increased at Mount Nourikura Neutron monitoring station. The likely one of the cause for such type of variation could be changing threshold cut off rigidity from $1.02 \mathrm{GV}$ to $1.15 \mathrm{GV}$ [9-12]. 


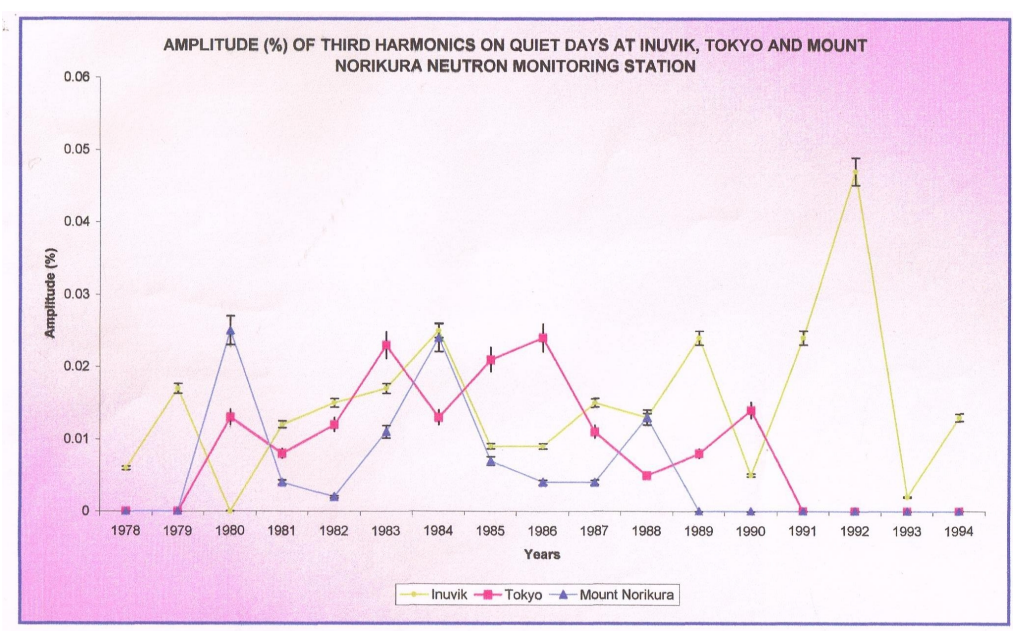

Figure 1

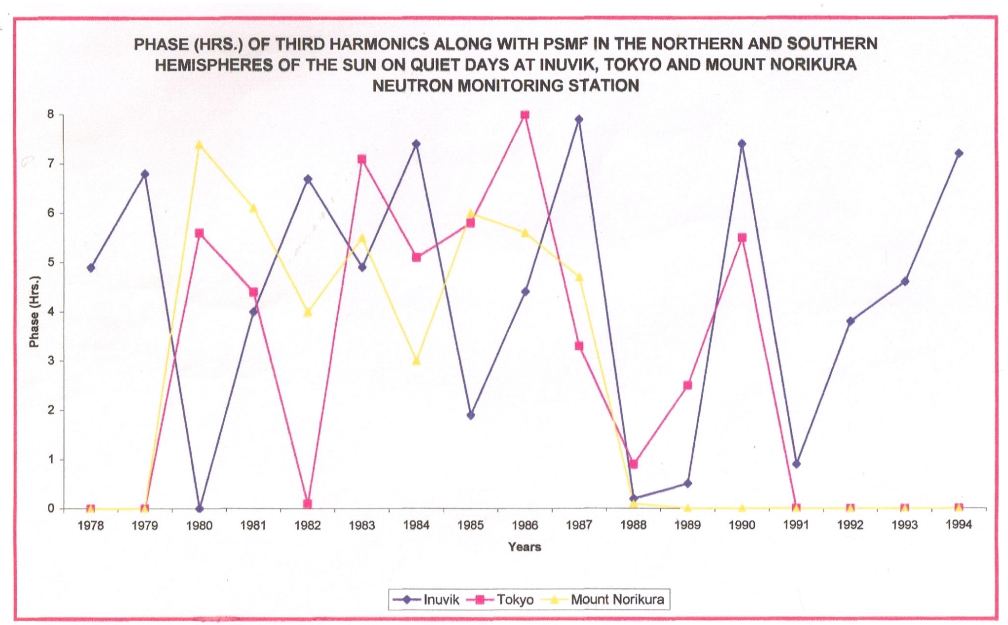

Figure 2

Further it is quite apparent from Fig. 1, that there is no systematic change in the amplitude of third harmonics on QDs. The amplitude of third harmonics of daily variation of QD is observed to be significantly low during 1981 as well as in 1990, which coincides with phase reversal of the solar poloidal magnetic filed [15]. Further, the amplitude of third harmonics on QD is low during 1985 and 1986, the year of minimum solar activity. The cause of such changes may be the increase of interplanetary magnetic field irregularities (IMF). The amplitude of third harmonics of daily variation on quiet days during 1978 and 1990 (Maximum Solar activity period of solar cycle 21 and 22) having same values. A clear 11 year type variation is observed at high latitude neutron monitoring station [14]. It is also observed from Fig. 2 that there is no systematic change in the phase of third harmonics of daily variation in cosmic ray intensity of QD. However, in the year 1990, the phase shifted to later hours, when the polarity of solar magnetic field in the northern hemisphere has changed from negative to positive. The polarity dependence of the phase shift change has been interpreted as a result due to the change of CR density distribution in space caused by the difference of CR drift motion in the positive and negative polarity state. 


\section{Acknowledgments}

The authors are indebtend to various experimental groups; in particular, Profs. M. Bercovitch, K. Nagashima and Miss Aoi Inoue for providing neutron monitor data.

\section{References}

[1] D. Venkatesan and Badruddin, Space Science Rev., 52, 121 (1990) and reference there in.

[2] Fujii, A., Nagashima, K., Fujimoto, K., Ueno, H. And Kondo, I. 1971, 12th ICRC, Hobart Tasmania, 2, 666.

[3] Ahluwalia, H. S. and Singh, S. 1973a, Proc. $13^{\text {th }}$ Int. Cosmic Ray Conf., Australia, $2: 948$.

[4] Ahluwalia, H. S. and Singh, S. 1973b, Proc. $13^{\text {th }}$ Int. Cosmic Ray Conf., Australia, 5:3129.

[5] Pomerantz, M. A. and Duggal, S. P. 1971, Space Sci. Rev., 12, 75.

[6] Rao, U. R. 1972, Space Sci. Rev., 12, 719.

[7] Agrawal, S. P. 1981, Journal Geophys. Res., 86: 10115.

[8] Kumar, S., Agrawal, R., Mishra, R. And Dubey, S. K. 2002, Bull Astronomical Soc. India, 30, 451.

[9] Shea, M. A. and Smart, D. F. 1983, 18th Int. Cosmic Ray Conf. Bangalore, 3:411.

[10] Smart, D. F. and Shea, M. A. 1987, 20th Int. Cosmic Ray Conf. Moscow, 4: 204.

[11] Shea, M. A. and Smart, D. F. 2001, 27th Int. Cosmic Ray Conf. Hemberg, 3: 4063.

[12] Kumar, S. Gulati, U., Khare, D. and Richharia, M. K. 1993, Bull Astronomical Soc. India, 21:395.

[13] Kumar, S., Richharia, M. K, Chauhan, M.L., Gulati, U., Khare, D. K. And Shrivastava, S. K. 1995, 24th Int. Cosmic Ray Conf. Italy, 4: 623.

[14] Richharia, M.K. 2011, $32^{\text {nd }}$ International Cosmic Ray Conference, Beijing China, 11, S.H. 3:4, 131.

[15] Richharia, M.K. 2011, 32 ${ }^{\text {nd }}$ International Cosmic Ray Conference, Beijing China, 11, S.H. 3:4, 127. 